\title{
Chromoblastomycosis, Overlooked and Undiagnosed: The Disease as a Mimic of other Neglected Tropical Diseases
}

\author{
Charmaine Vanessa S. Chamberlin, ${ }^{1}$ Maria Christina Filomena R. Batac ${ }^{1}$ and Eileen Liesl A. Cubillan ${ }^{2}$ \\ ${ }^{1}$ Section of Dermatology, Department of Medicine, Philippine General Hospital, University of the Philippines Manila \\ ${ }^{2}$ Section of Dermatology, Department of Medicine, College of Medicine and Philippine General Hospital, University of the Philippines Manila
}

\begin{abstract}
Chromoblastomycosis is an endemic mycoses which has been misdiagnosed or underdiagnosed in the past. This may be due to the clinicopathologic characteristics that it shares with other neglected tropical diseases such as leprosy and cutaneous tuberculosis. Correlating clinical findings with histopathologic cues will lead clinicians to correct diagnosis and subsequent treatment success.
\end{abstract}

Key Words: chromoblastomycosis, chronic, invasive fungal infections, neglected tropical diseases, itraconazole

\section{INTRODUCTION}

Chromoblastomycosis is a chronic fungal infection of cutaneous and subcutaneous tissues. It is characterized by slow growing localized nodular or verrucous lesions and is diagnosed through histopathology and fungal culture. There have been many reports of chromoblastomycosis around the world, with most incidences occurring in countries bordering on the equator. ${ }^{1}$ The actual prevalence is unknown but usually affected individuals are from poor and remote communities of low-income tropical countries. This prompted its recent addition to the World Health Organization list of neglected tropical diseases. Like other neglected tropical diseases such as leprosy, chromoblastomycosis also presents with granulomatous changes on histopathology, differentiated through the presence of muriform cells. Here we present a case of chromoblastomycosis in the Philippines previously diagnosed and unsuccessfully treated as lepromatous leprosy and cutaneous tuberculosis.

\section{CASE REPORT}

A 58-year-old male jeepney driver, presented with a 20-year history of verrucous lesions on the right foot. These lesions evolved following a bicycle accident where

Corresponding author: Charmaine Vanessa S. Chamberlin, MD Section of Dermatology Department of Medicine Philippine General Hospital University of the Philippines Manila Taft Avenue, Manila 1000, Philippines

Telephone: +6325548400 local 5105/5106

Email: charm.chamberlin@gmail.com the patient sustained abrasions on the lateral aspect of the right foot. Multiple consultations were done for the frequent infections on the increasing number of lesions without improvement until four years prior when a biopsy was performed and signed out as lepromatous leprosy. The patient underwent multi-drug treatment with rifampicin, clofazimine, and dapsone for 6 months without 
improvement. A repeat biopsy was done and the patient was treated for cutaneous tuberculosis with a regimen composed of isoniazid, rifampicin, pyrazinamide, and ethambutol for 1 year. Despite undergoing two different treatment regimens, there was still an increase in the number of lesions and frequent infections.

Examination upon consultation yielded multiple well demarcated erythematous and hyperpigmented verrucous plaques with filiform projections and scales on the surface laced with seropurulent discharge and hemorrhagic crusts covering almost the entire dorsum of the right foot extending toward the ankle and lower leg (Figures 1 and 2).

The patient had no other comorbidities and the rest of the physical examination was unremarkable. The histopathlogical examination showed granulomatous changes with lymphocytes (Figure 3), and brown multinucleated giant cells with sclerotic bodies or the muriform cells characteristic of chromoblastomycosis (Figure 4).

A slit skin smear, tissue bacterial culture and sensitivity, tissue $\mathrm{TB}$ culture and sensitivity, and PCR were done to rule out previous diagnoses and all tests were negative.

A repeat fungal culture showed growth of velvety olivaceous fungi (Figure 5) yielding dematiaceous fungi on mounting; a specific species, however, could not be identified. Itraconazole $200 \mathrm{mg}$ daily for a minimum of 6 months was given to this patient with significant response.
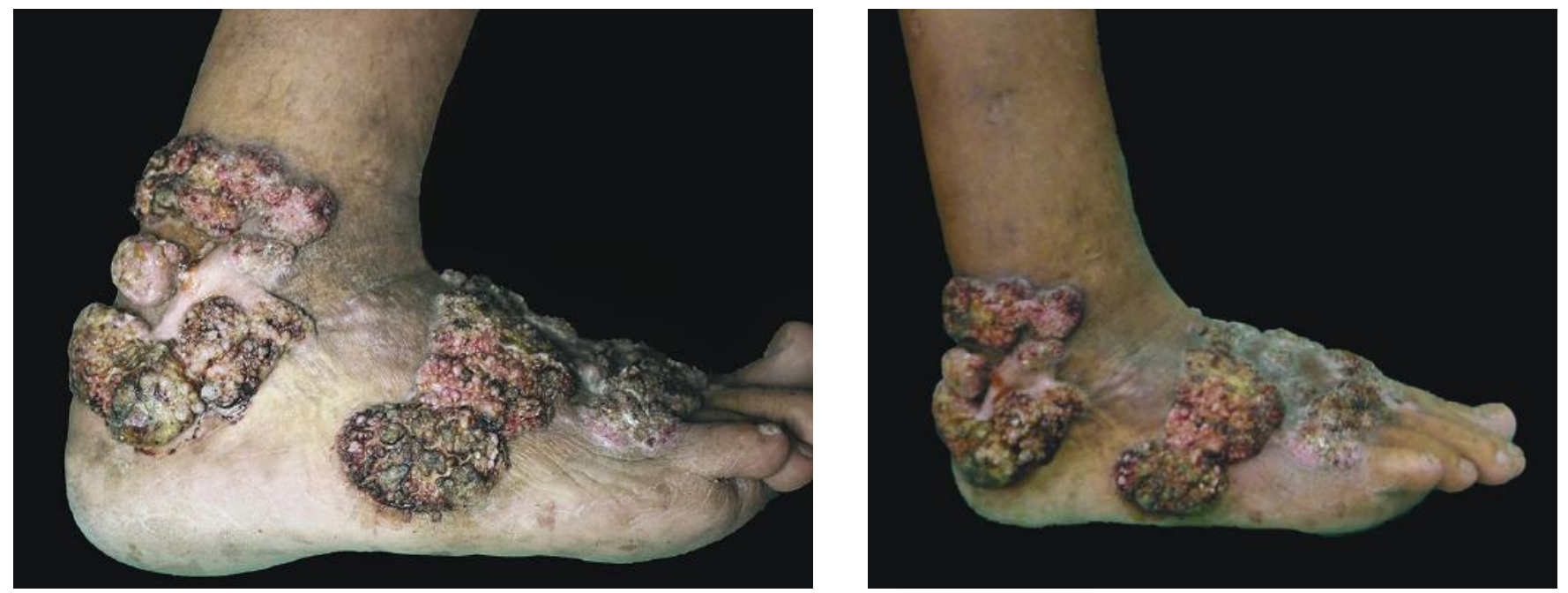

Figure 1. Cutaneous lesions consisting of well-defined erythematous and hyperpigmented plaques and tumors with papillomatous surface covered with brown and yellow-colored crusts found on the right ankle area, on the area of the lateral malleolus, dorsolateral extending to the dorsomedial area of the right foot.
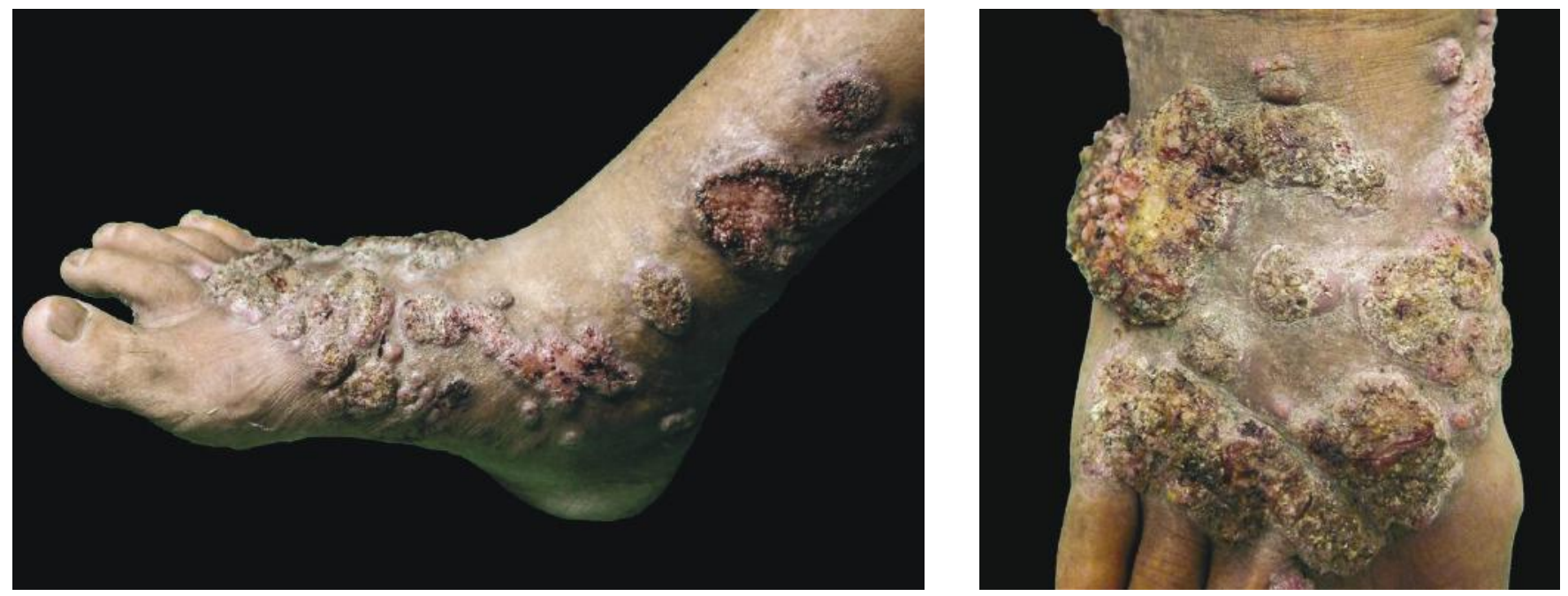

Figure 2. Images show multiple areas of ulceration, fissuring, and erosions within the plaques, nodules, and tumors on the dorsum to the dorsomedial aspect of the right foot extending toward the medial lower leg. The ulcerated areas are noted to be draining seropurulent discharge and have areas of brown and honey-colored crust. 

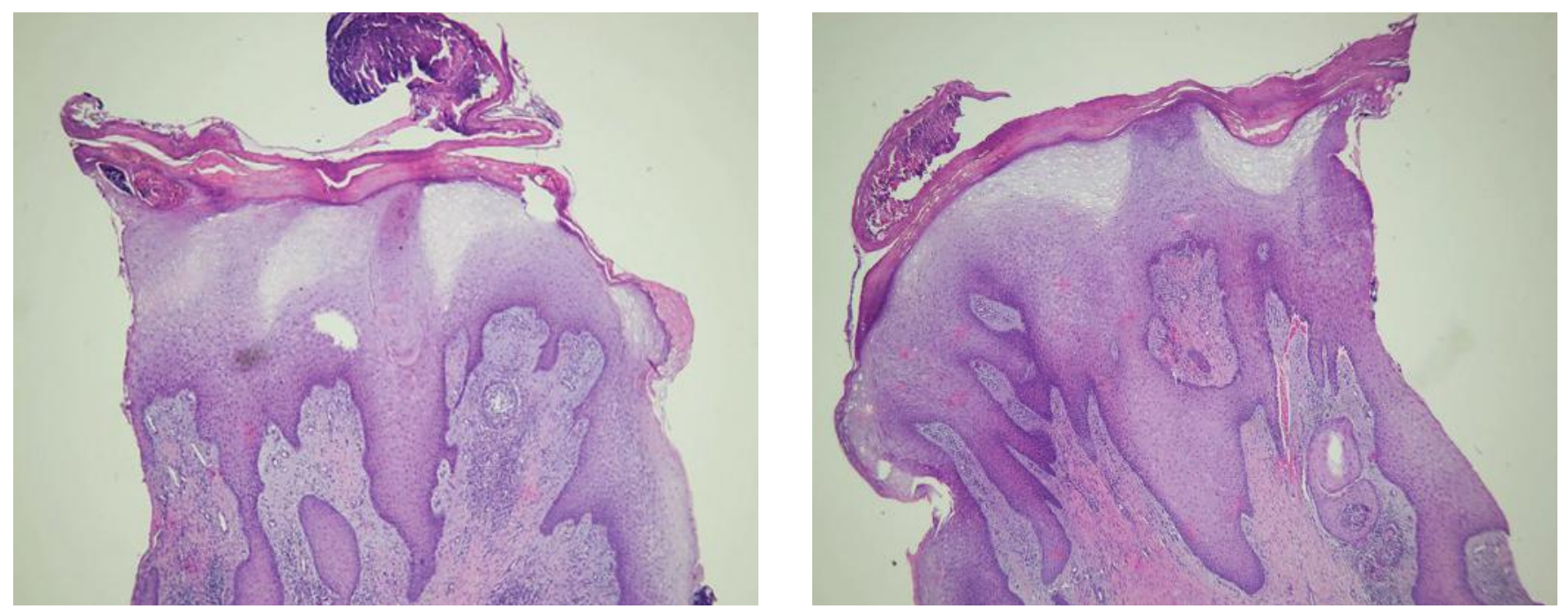

Figure 3. Scanning view of $4 \mathrm{~mm}$ punch biopsy sections showing parakeratosis, spongiosis and pseudocarcinomatous hyperplasia.
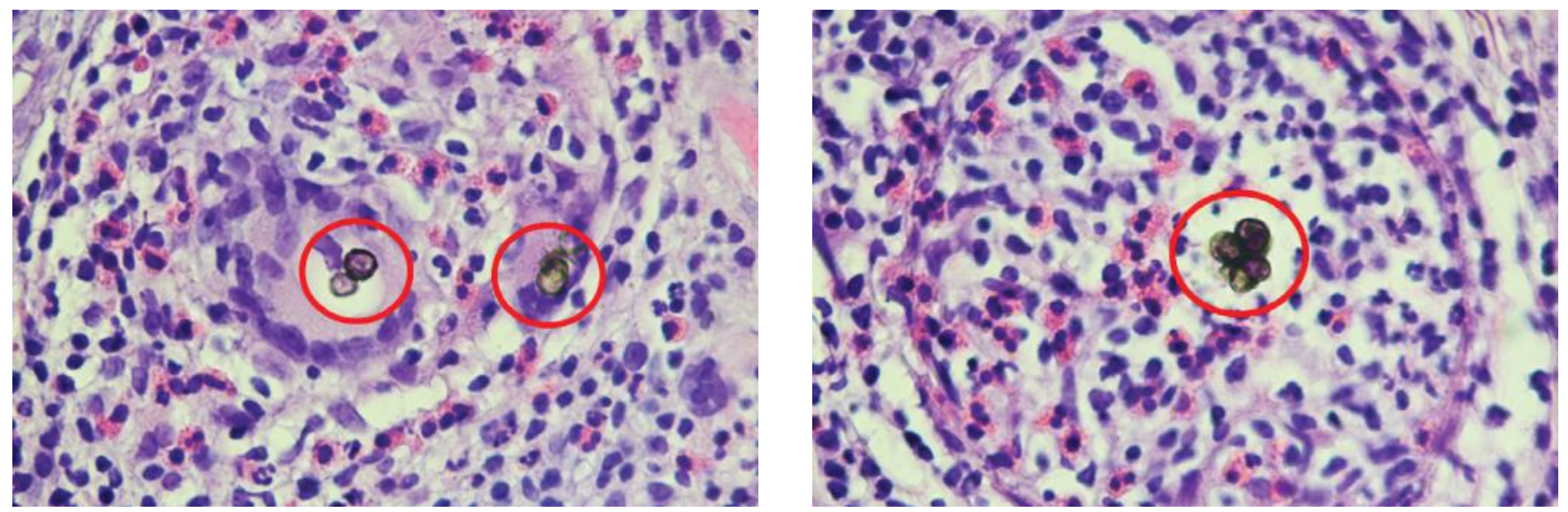

Figure 4. Higher magnification showing multinucleated giant cells with sclerotic bodies also known as muriform cells commonly described as "copper pennies".
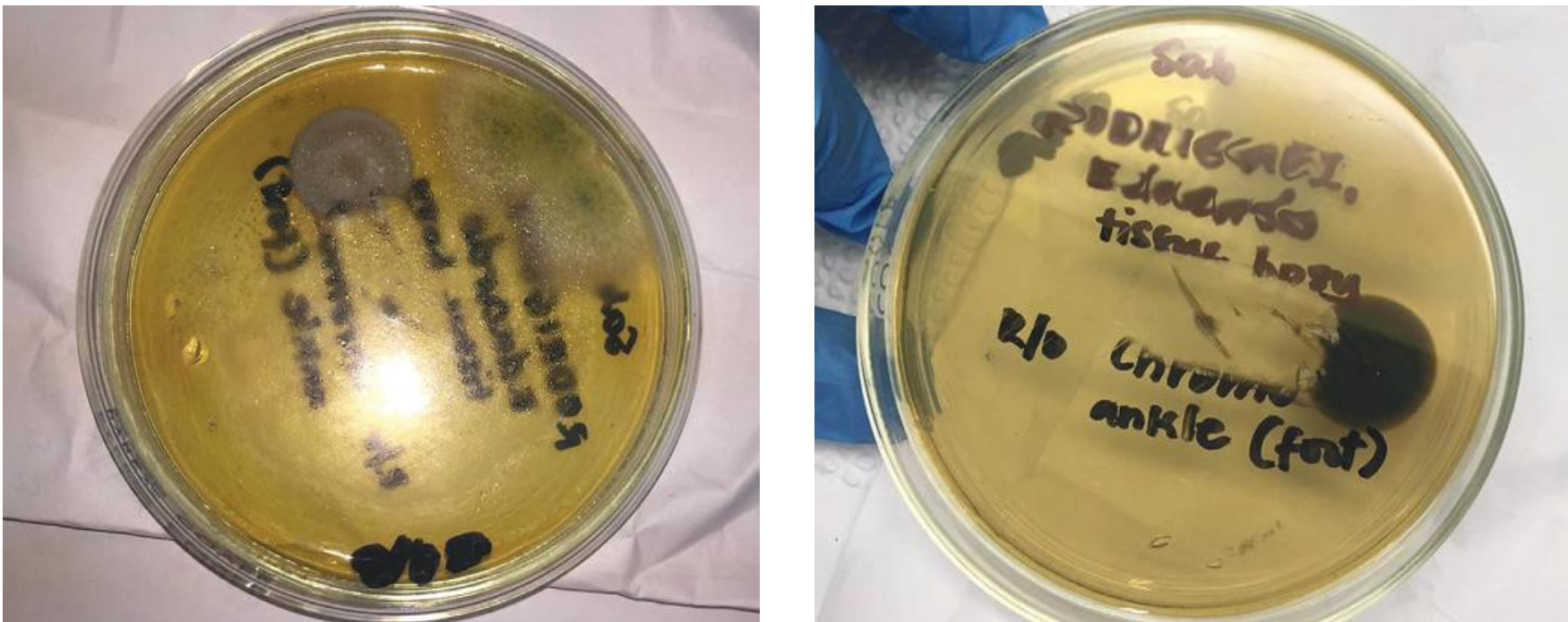

Figure 5. Images show topside of fungal culture colony growth on SDA with velvety, grey-olivaceous colonies, underside shows dark green-black colored colony suggesting dematiaceous (melanized) fungi. 


\section{DISCUSSION}

Chromoblastomycosis is one of the most common mycoses caused by melanized or dematiaceous fungi wherein fungal elements are deposited through a break in the skin, usually through a traumatic event. ${ }^{1,2}$ Inoculation and infection usually causes a chronic granulomatous response in the tissues affected and the host may be immunocompetent but still be unable to clear the infection..$^{1,2}$ The progress of the lesions are slow, however chronicity will show a more characteristic appearance defined as exophytic, verrucous, and wart-like with cauliflower appearing projections which are hyperpigmented and crusted., ${ }^{1,2}$ Secondary bacterial infections are common and this may lead to ulcerations draining foul-smelling seropurulent discharge. ${ }^{1}$

In a comprehensive summarization of the case reports of chromoblastomycosis around the world by Queiroz-Telles et al. in 2016, majority of the cases were reported from Latin America and the Caribbean. Other areas of prevalence are in tropical and subtropical regions of the planet, specifically in between the latitudes of $30^{\circ}$ North and $30^{\circ}$ South. ${ }^{2}$ According to the data accumulated by the Philippine Dermatological Society between the years 2011-2017, there have been 10 documented cases of chromoblastomycosis. The most number of cases were diagnosed in 2013 which were documented in 3 male patients. Out of the 10 documented cases, 6 were male and 4 were female with an age distribution of 35-69 years old. ${ }^{3}$ Two cases have been reported locally showing the causative agent as Fonsecaea compacta. ${ }^{4}$

The most common etiological agents of chromoblastomycosis are melanized fungi related to black yeasts and these include Fonsecaea pedrosoi, Fonsecaea monophora, Cladophialophora carrionii, Rbinocladiella aquaspera and occasional Phialophora and Exophalia species. ${ }^{1,2,4}$ All of these are members of the order Chaetothyriales and the family Herpotrichiellaceae. ${ }^{1,2}$ On fungal culture, the causative organisms usually present with felt-like, gray-olivaceous colonies with regular, melanized, branched hyphae. The species are differentiated through the conidiophores which are the spore like projections at the end of the branched hyphae. ${ }^{1,2,5}$ What is common among them, aside from the black and brown pigment are the unique morphological and cellular plasticity that they manifest. Upon infection, celldifferentiation occurs and this leads to isodiametric swelling and cross-septation. These changes form the muriform cells or the multinucleated giant cells with sclerotic bodies typically described as "copper pennies" upon histopathologic examination.., 6 These muriform cells are directly associated with a marked granulomatous response with parakeratosis, pseudoepitheliomatous or pseudocarcinomatous hyperplasia, spongiosis, and increased lymphocytes. . $, 2,5,7,8$

Given the characteristic histopathological findings of muriform cells embedded in a granulomatous background, this leads to the scrutiny of misdiagnosis as leprosy and cutaneous tuberculosis. These diseases both present a granulomatous response with dense infiltrates and presence of lymphocytes. Cutaneous tuberculosis present with epidermal hyperplasia and initially a dense neutrophilic infiltrate which later present with necrotizing granulomatous inflammation surrounded by numerous lymphocytes in chronic infections. ${ }^{8}$ Similarities among chronic granulomatous infections are common which stresses the importance of correlation with history and physical examination. It allows for the dermatologist and the dermatopathologist to have an idea what to look for, such as the case of muriform cells in chromoblastomycosis, evident in the skin biopsy of our patient.

A criteria of cure was established by Bonifaz, Solis, and Saul in 2016. Cure needs to be documented clinically, mycologically, and histopathologically. These are indicated by apparent resolution of lesions, disappearance of granulomatous reaction and muriform cells on biopsy, and negative fungal cultures. ${ }^{9}$

Treatment options for chromoblastomycosis include excision surgery, which is currently the best physical method for complete clinical cure in small well-demarcated lesions. ${ }^{10}$ Cryotherapy at -196 degrees Celsius at 30 seconds to 4 minutes is recommended for small lesions or lesions that have regressed with previous anti-fungal treatment. ${ }^{10,11}$ Laser therapy has previously been done with a $\mathrm{CO}_{2}$ laser at 10,600 $\mathrm{nm}$ wavelength for ablation, however it is still not routinely used. ${ }^{12}$ Heat therapy or the application of disposable chemical pocket warmers to affected sites have shown promising responses due to the inability of the causative organism to grow beyond 40 degree Celsius. Despite the promising quality of this treatment option, further investigation is warranted. ${ }^{13}$ Though physical methods of treatment were found to be effective adjuncts to treatment, anti-fungals are still at the forefront to achieving cure. ${ }^{1,9,10}$

There are many different studies on the anti-fungal susceptibility of the causative agents of chromoblastomycosis. Despite not being conclusive, the use of itraconazole and/or terbinafine as treatment have been shown to be effective. . $2,9,10^{2}$ The current best practice was indicated to be itraconazole and terbinafine either as single or combined, given in high doses at 6-12 month periods. ${ }^{9}, 10$ Giving the oral anti-fungals with concurrent cryosurgery may be done or if chemical heat packs are available, these may be an adjunct to treatment as well. ${ }^{13}$

The patient's initial treatment with $100 \mathrm{mg} / \mathrm{capsule}$ of itraconazole at 2 capsules daily for 2 months showed a significant improvement in the appearance of the lesions. Within 2 months, there was noted resolution of the ulcerations and seropurulent discharge. Aside from this, there was a decrease in the thickness of the plaques and regression in size of the nodules. Most lesions had lost the hyperpigmented papillary and cauliflower surface and had transformed to flat pink plaques with some fine white scaling on the surface (Figure 6). Other lesions had become hyperpigmented patches. The patient reported disappearance of pain and pruritus. 

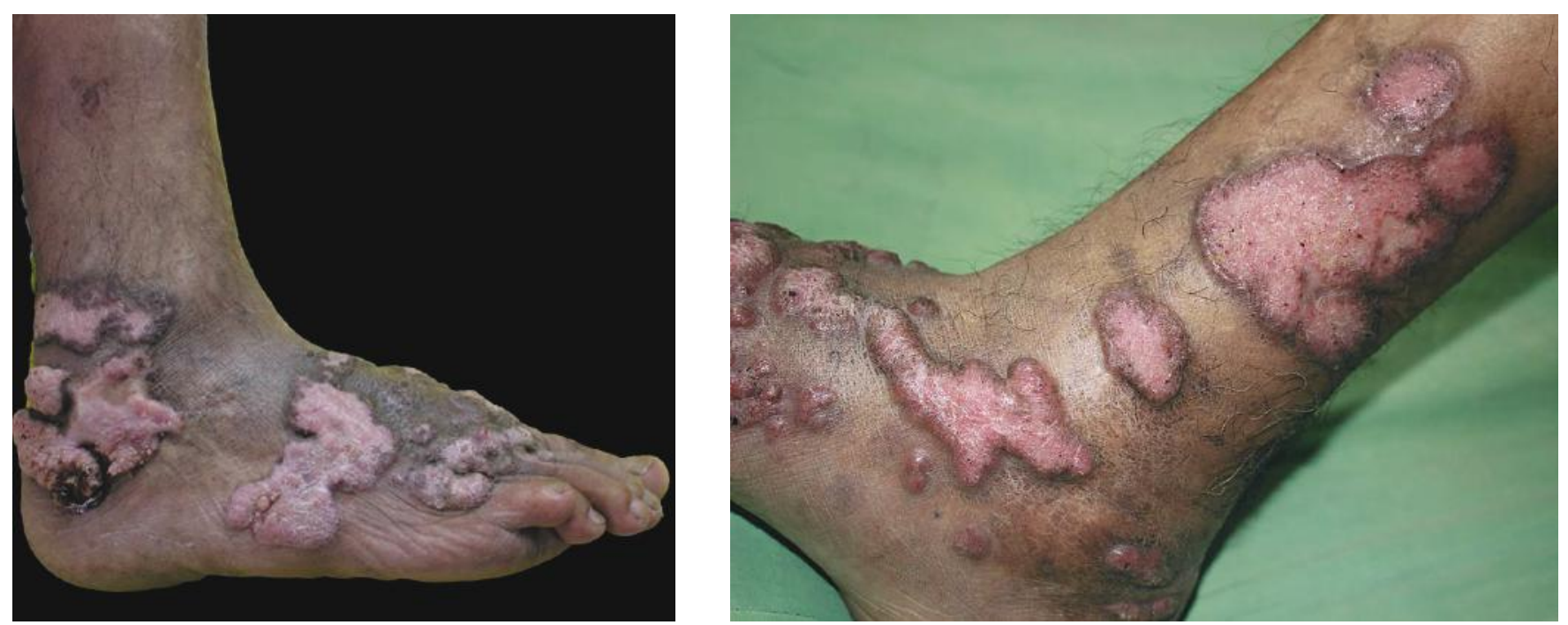

Figure 6. Images taken after 2 months of itraconazole 100mg/capsule 2 capsules daily treatment with notable decrease in thickness and hyperpigmented cauliflower-like projections on the surface of the plaques and nodules. Resolution of ulcers replaced by dry erythematous plaques with hyperpigmented borders.

\section{CONCLUSION}

Chromoblastomycosis is a neglected endemic mycoses which may be misdiagnosed or underdiagnosed as other neglected tropical diseases leading to unsuccessful treatment. Verrucous lesions in tropical countries should always be correlated clinically to the histopathologic findings. Neglected tropical diseases may present with similar histopathologic findings; being knowledgeable on the specific clinical and histopathologic characteristics will help differentiate diagnosis which will then allow the physician to provide options to the patients on a treatment regimen that will give the highest chance of cure.

\section{Disclaimer}

The views expressed in this article are the authors own and do not reflect the views of the institution.

\section{Statement of Authorship}

All authors approved the final version submitted.

\section{Author Disclosure}

All authors declared no conflict of interest.

\section{Funding Source}

This paper was funded by the authors. No external funding agency.

\section{REFERENCES}

1. Queiroz-TellesF,deHoog S,SantosDWCL,etal.Chromoblastomycosis. Clin Microbiol Rev. 2017;30:233-76.

2. Queiroz-Telles F, Fahal AH, Falci DR, Caceres DH, Chiller T, Pasqualotto AC. Neglected endemic mycoses Lancet Infect Dis. 2017;17(11):e367-77. doi: 10.1016/S1473-3099(17)30306-7.

3. Philippine Dermatological Society Health Information Systems Statistics from January 2011 until November 2017.

4. Batac MCR, Denning D. Serious fungal infections in the Philippines. Eur J Clin Microbiol Infect Dis. 2017 ;36(6):937-41. doi: 10.1007/s10096-017-2918-7.

5. Minotto R, Edelweiss MIA, Scroferneker ML. Study on the organization of cellular elements in the granulomatous lesion caused by chromoblastomycosis. J Cutan Pathol. 2017;44(11):915-8. doi: 10.1111/cup.13014.

6. Yap FBB. Chromoblastomycosis. Int J Infect Dis. 2010 Jun;14(6):e543-4. doi: 10.1016/j.ijid.2009.07.005.

7. Skupsky H, Junkins-Hopkins J. Counterfeit Pennies: Distinguishing Chromoblastomycosis From Phaeohyphomycotic Infections. Am J Dermatopathol. 2017 ;39(6):485-7. doi: 10.1097/ DAD.0000000000000679

8. Calonje E, Brenn T, Lazar A, McKee P. McKees pathology of the skin, 4th Ed. Elsevier, Saunders;2011.

9. Bonifaz A, Paredes-Solís V, Saúl A. Treating chromoblastomycosis with systemic antifungals. Expert Opinion on Pharmacotherapy. 2004;5(2):247-54. doi:10.1517/14656566.5.2.247.

10. Ameen M. Chromoblastomycosis: clinical presentation and management. Clin Exp Dermatol. 2009;34(8):849-54. doi:10.1111/ j.1365-2230.2009.03415.x.

11. Castro LG, Pimentel ER, Lacaz CS. Treatment of chromomycosis by cryosurgery with liquid nitrogen: 15 years' experience. Int J Dermatol. 2003; 42: 408-12.

12. Tsianakas A, Pappai D, Basoglu Y, et al. Chromomycosis - successful $\mathrm{CO}_{2}$ laser vaporization. J Eur Acad Dermatol Venereol. 2008;22(11): 1385-6, doi:10.1111/j.1468-3083.2008.02649.x.

13. Hiruma M, Kawada A, Yoshida M, Kouya M. Hyperthermic treatment of chromomycosis with disposable chemical pocket warmers. Report of a successfully treated case, with a review of the literature. Mycopathologia. 1993;122:107-14. 